\title{
Nineteen-port photonic lantern with multimode delivery fiber
}

Noordegraaf, Danny; Skovgaard, Peter M. W.; Sandberg, Rasmus Kousholt; Maack, M. D.; BlandHawthorn, J.; Lawrence, J. S.; Lægsgaard, Jesper

\section{Published in:}

Optics Letters

Link to article, DOI:

10.1364/OL.37.000452

Publication date:

2012

Document Version

Publisher's PDF, also known as Version of record

Link back to DTU Orbit

Citation (APA):

Noordegraaf, D., Skovgaard, P. M. W., Sandberg, R. K., Maack, M. D., Bland-Hawthorn, J., Lawrence, J. S., \& Lægsgaard, J. (2012). Nineteen-port photonic lantern with multimode delivery fiber. Optics Letters, 37(4), 452454. https://doi.org/10.1364/OL.37.000452

\section{General rights}

Copyright and moral rights for the publications made accessible in the public portal are retained by the authors and/or other copyright owners and it is a condition of accessing publications that users recognise and abide by the legal requirements associated with these rights.

- Users may download and print one copy of any publication from the public portal for the purpose of private study or research.

- You may not further distribute the material or use it for any profit-making activity or commercial gain

- You may freely distribute the URL identifying the publication in the public portal 


\title{
Nineteen-port photonic lantern with multimode delivery fiber
}

\author{
D. Noordegraaf, ${ }^{1,2, *}$ P. M. W. Skovgaard, ${ }^{1}$ R. H. Sandberg, ${ }^{1}$ M. D. Maack, ${ }^{1}$ \\ J. Bland-Hawthorn, ${ }^{3,4}$ J. S. Lawrence, ${ }^{5,6}$ and J. Lægsgaard ${ }^{2}$ \\ ${ }^{1}$ NKT Photonics A/S, Blokken 84, DK-3460 Birkerø d, Denmark \\ ${ }^{2}$ DTU Fotonik, Technical University of Denmark, DK-2800, Denmark \\ ${ }^{3}$ School of Physics, University of Sydney, New South Wales 2006, Australia \\ ${ }^{4}$ Institute of Photonics and Optical Sciences, University of Sydney, New South Wales 2006, Australia \\ ${ }^{5}$ Australian Astronomical Observatory, Epping, New South Wales 2121, Australia \\ ${ }^{6}$ Department of Physics and Astronomy, Macquarie University, New South Wales 2109, Australia \\ *Corresponding author: dno@nktphotonics.com
}

Received November 14, 2011; accepted December 12, 2011;

posted December 14, 2011 (Doc. ID 158202); published February 3, 2012

\begin{abstract}
We demonstrate efficient multimode (MM) to single-mode (SM) conversion in a 19-port photonic lantern with a $50 \mu \mathrm{m}$ core MM delivery fiber. The photonic lantern can be used within the field of astrophotonics for coupling MM starlight to an ensemble of SM fibers in order to perform fiber-Bragg-grating-based spectral filtering. An MM delivery fiber spliced to the photonic lantern offers the advantage that the delivery fiber guides the light from the focal plane of the telescope to the splitter. Therefore, it is no longer necessary to have the splitter mounted directly in the focal plane of the telescope. The coupling loss from a $50 \mu \mathrm{m}$ core MM fiber to an ensemble of 19 SM fibers and back to a $50 \mu \mathrm{m}$ core MM fiber is below $1.1 \mathrm{~dB}$. (C) 2012 Optical Society of America

OCIS codes: $\quad 060.2340,350.1260$.
\end{abstract}

Multimode (MM) to single-mode (SM) converters or photonic lanterns were developed for the field of astrophotonics to realize fiber-Bragg-grating-based filtering in connection with MM optical fibers. Traditionally, MM fibers have been used to transport light from the telescope to the optical spectrograph. These large-core MM fibers are preferred in order to increase the amount of collected light $[\underline{1}, 2]$. Photonic lanterns that efficiently couple light from a large-core MM fiber to an ensemble of SM fibers allows for the realization of revolutionary new instruments. These new instruments include $\mathrm{OH}$ suppression of the hundreds of extremely bright, ultranarrow emission lines in the IR part of the spectrum, that originate from high-altitude hydroxyl in the Earth's atmosphere. The photonic lanterns couple light from an MM fiber to an ensemble of SM fibers, where identical fiber Bragg gratings filter out the $\mathrm{OH}$ emission lines [3,4]. In the end, another photonic lantern combines the light from the SM fiber ensemble back into an MM fiber that feeds the optical spectrograph. Another promising instrument enabled by the photonic lantern is the photonic integrated multimode microspectrograph [5]. In this case the photonic lantern delivers an SM input for an arrayed waveguide grating that disperses the light. This concept potentially enables fabrication of compact and cheap spectrographs.

The principle of the photonic lantern was first demonstrated in 2005 by Leon-Saval et al. [6], and thereafter low-loss photonic lanterns with 7 and $\overline{6} 1 \mathrm{SM}$ ports have been demonstrated $[7,8]$. None of these low-loss devices featured an MM delivery fiber. Recently, two new approaches have been demonstrated that enable the fabrication of compact photonic lanterns. These are a tapered multicore fiber approach [9] and direct inscription of the photonic lanterns in integrated waveguides with ultrafast lasers $[\underline{10}, \underline{11}]$.

In this Letter we demonstrate, to the best of our knowledge, the first low-loss photonic lanterns with MM deliv- ery fibers, and an investigation of how the NA coupled into the devices influences the transmission loss. The purpose of the delivery fiber is in one end to guide the starlight from the focal plane of the telescope to the photonic lantern and in the other end to guide the light from the photonic lantern to the spectrograph. This allows for a more robust system, where the photonic lanterns can be packaged and isolated away from the optomechanical interfaces of the telescope and the spectrograph. Crucially, this allows the telescope delivery fibers to be optically interfaced to micro-optics placed at the telescope focal plane and allows the spectrograph delivery fibers to be installed in close contact in a V-groove array that forms the spectrograph slit.

Figure 1 shows an illustration of the fabricated photonic lantern. A bundle of 19 Corning SMF-28 fibers are inserted into a low refractive index glass capillary tube. These SM fibers have a higher order mode (HOM) cutoff wavelength at $1260 \mathrm{~nm}$ and a diameter of the core, cladding, and coating of $8.2,125$, and $250 \mu \mathrm{m}$, respectively. The fiber-filled capillary tube is fused into a solid glass element and tapered to a $50 \mu \mathrm{m}$ core MM waveguide. The core of the waveguide consists of the tapered SM fibers, and the cladding is formed by the surrounding low-index capillary tube. The fused bundle is tapered by a factor of 11 in diameter over a length of $60 \mathrm{~mm}$.

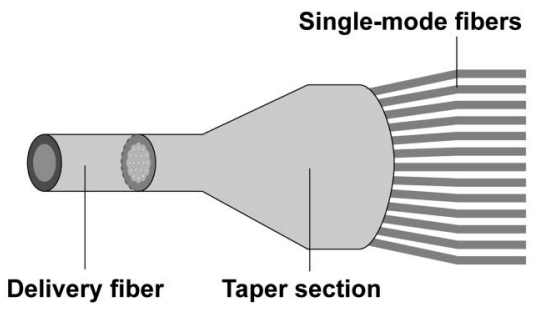

Fig. 1. Schematic illustration of the photonic lantern. 


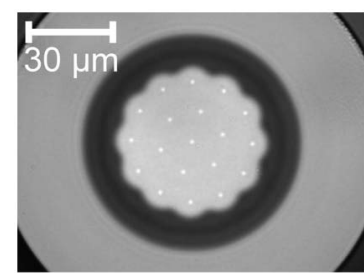

(a)

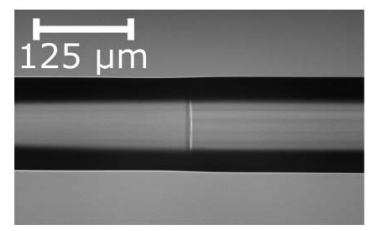

(b)

Fig. 2. (a) Microscope image of the cross section of the tapered MM tip of the photonic lantern. (b) Splice between the tapered MM tip of the photonic lantern (right side) and the $50 \mu \mathrm{m}$ core MM fiber.

Figure 2(a) shows the tip of the fused tapered fiber bundle. The bundle tip is spliced to a step-index MM fiber with a core diameter of $50 \mu \mathrm{m}$ and an NA of 0.22 (Fiberguide Industries). A $5 \mathrm{~m}$ long length of MM fiber is used, ensuring that the fiber can reach the focal plane feed optics of a telescope. Figure 2(b) shows an image of the splice. The fusing, tapering, and splicing are performed on a filament-based glass processing station from Vytran [12].

Low-loss coupling of light from the MM fiber into the SM fiber ensemble may be achieved if the number of degrees of freedom in the SM fiber ensemble is equal to or higher than in the MM waveguide [13]. Therefore, efficient coupling or transformation of modes from the MM waveguide to the SM fiber ensemble is possible, when the number of excited modes in the MM waveguide is less than or equal to the number of SM ports. For a step-index fiber, the number of supported modes $M$ in one polarization orientation is calculated from the $V$ parameter [14]

$$
M \approx \frac{V^{2}}{4} .
$$

The $V$ parameter for a step-index fiber is defined as

$$
V=\frac{2 \pi}{\lambda} a \mathrm{NA},
$$

where $\lambda$ is the free space optical wavelength, $a$ is the radius of the waveguide core, and NA is the numerical aperture. By rearranging Eqs. (1) and (2), it is found that the approximate NA of the light in an MM fiber where the first $M$ number of modes are excited is given by

$$
\mathrm{NA} \approx \frac{\lambda \sqrt{M}}{\pi a} .
$$

At a wavelength of $1.53 \mu \mathrm{m}$ and for a core diameter of $50 \mu \mathrm{m}$, the 19 lowest order modes have an NA of $\sim 0.085$. The core diameter and NA are chosen to sufficiently sample the seeing-limited telescope point spread function for the $4 \mathrm{~m}$ diameter Anglo-Australian Telescope [15].

Three photonic lanterns were fabricated. Incoherent light from an ASE source centered at $1530 \mathrm{~nm}$ and with a $10 \mathrm{~dB}$ width of $40 \mathrm{~nm}$ is in turn spliced to each of the SM fiber ports of the photonic lanterns. The SM to MM transmission loss and the NA of the light out of the MM fiber is then measured for each port. The transmitted loss is measured using an integrating sphere, and the NA is measured by scanning the far field. Table 1 shows the average measured loss and NA and the highest measured value for a single port in brackets.

Low loss from SM to MM is measured for all three fabricated devices. This is expected, because the degrees of freedom in the MM fiber are higher than in the SM fiber ensemble. The output NA matches well to the calculated NA of exciting the 19 lowest order modes.

Three devices are fabricated such that two devices, \#1 and \#2, can be spliced back to back. Thereby, the MM to SM to MM transmission loss of a system with two photonic lanterns can be measured. Device \#3 is used as an input source for coupling light into the two devices. For such a system to work, it is important that the NA of the light is maintained through the $5 \mathrm{~m}$ long $\mathrm{MM}$ delivery fiber of device \#1. NA upconversion is due to mode coupling to HOMs of the optical fiber and is caused by scattering or translation variance of the fiber, i.e., micro bends, fiber imperfections, or diameter fluctuations. Coupling to modes higher than the 19 lowest order modes will result in a loss in the photonic lantern.

In order to investigate that the MM fiber preserves NA, light from the $1530 \mathrm{~nm}$ ASE source delivered in an SMF28 fiber is coupled into the MM fiber. The light coupled in has an NA of 0.106 . Figure 3 shows the measured transmission loss and the NA out of the fiber at different coil diameter. The figure shows that the NA is well preserved down to a coil diameter of around $5 \mathrm{~cm}$, where some of the high NA light is lost. Transmission loss increases around a coil diameter of $7 \mathrm{~cm}$.

The MM to SM to MM transmission loss of the photonic lanterns is measured by splicing the 19 SM fibers of device \#1 randomly to the 19 SM fibers of device \#2. The loss is measured by launching light with a $1530 \mathrm{~nm}$ ASE source into the MM fiber of device \#1 and measuring the power out of the MM fiber of device \#2 with an integrating sphere. Figure 4 shows the measured loss at different launch NAs. The different launch NAs are obtained by NA filtering of the light source using step-index fibers with different mode field diameters (square points) and

Table 1. Average SM to MM Transmission Loss and Average NA of the Light out of the MM Delivery Fiber (Values in Brackets Show the Highest Measured Value for a Single Port)

\begin{tabular}{llll}
\hline & \multicolumn{1}{c}{ Device \#1 } & \multicolumn{1}{c}{ Device \#2 } & Device \#3 \\
\hline SM to MM loss (dB) & $0.18(0.36)$ & $0.16(0.27)$ & $0.05(0.07)$ \\
NA 95\% power limit & $0.097(0.108)$ & $0.107(0.114)$ & $0.095(0.109)$ \\
\hline
\end{tabular}




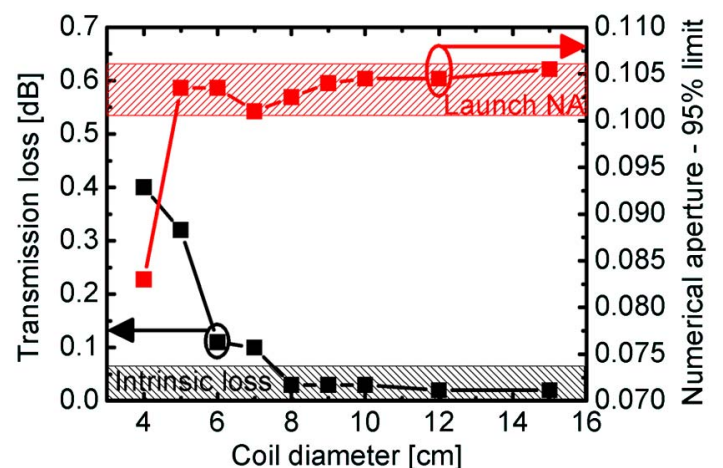

Fig. 3. (Color online) Transmission loss and NA dependence on the coiling diameter of the $5 \mathrm{~m}$ long, $50 \mu \mathrm{m}$ core MM fiber.

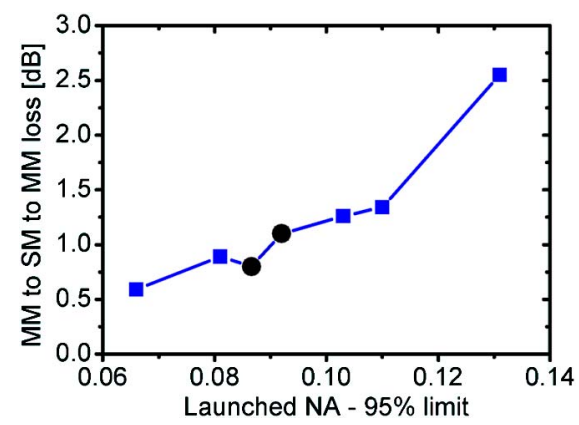

Fig. 4. (Color online) MM to SM to MM transmission loss through two photonic lanterns spliced back to back (devices $\# 1$ and \#2) as a function of the launched NA. The measurement is made with an ASE source centered at $1530 \mathrm{~nm}$.

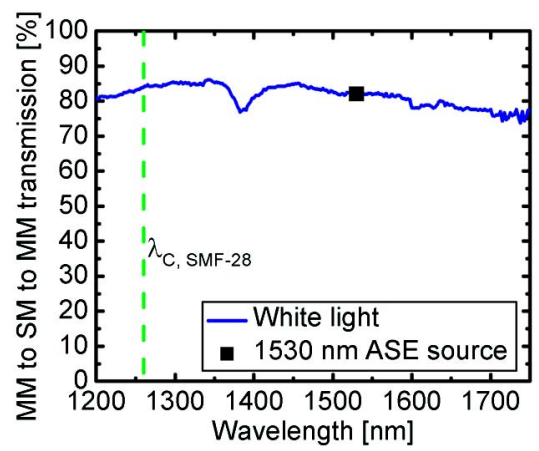

Fig. 5. (Color online) Spectral transmission through two photonic lanterns spliced back-to-back (solid blue line). The transmission measured with a $1530 \mathrm{~nm}$ ASE source is indicated by the red square. The dashed green line indicates the HOM cutoff wavelength of the SMF-28 fibers.

with the photonic lantern device \#3 (circle points). From low to high, the NA is obtained with the following: $14 \mu \mathrm{m}$ core fiber, $10 \mu \mathrm{m}$ core fiber, low-NA port of device \#3 $(\mathrm{NA}=0.087)$, high-NA port of device \#3 $(\mathrm{NA}=0.092)$, SMF-28 fiber, HI1060 fiber, and UHNA4 fiber. A loss below $1.1 \mathrm{~dB}$ is measured when the launched NA is below 0.092 .
The transmission loss of the two photonic lanterns in the back-to-back configuration is also characterized as a function of wavelength. This is done with the low-NA port of device \#3 as the input and replacing the powermeter with an optical spectrum analyzer and the ASE source with a white light source. Figure 5 shows the measured transmission (solid blue line). The transmission measurement made with the ASE source is indicated by the square, and the cut-off wavelength for HOMs is indicated by the green dashed line. Transmission through the two devices is flat. A dip in the transmission is observed at a wavelength of $1385 \mathrm{~nm}$, which is attributed to the water absorption in the fibers.

In conclusion, we have fabricated and characterized 19-port photonic lanterns with $50 \mu \mathrm{m}$ core MM delivery fibers. A system of two photonic lanterns spliced back to back provides efficient conversion from an MM fiber into a 19 SM fiber ensemble and back into an MM fiber, provided that the launched NA into the devices is $<0.092$. This coupling is done with a total transmission loss of $<1.1 \mathrm{~dB}$ at a wavelength of $1530 \mathrm{~nm}$. Furthermore, it is demonstrated that the transmission spectrum is flat in the spectral region from $1200 \mathrm{~nm}$ to $1750 \mathrm{~nm}$.

\section{References}

1. S. C. Barden, L. W. Ramsey, and R. J. Truax, Publ. Astron. Soc. Pac. 93, 154 (1981).

2. P. M. Gray, Proc. SPIE 374, 160 (1983).

3. J. Bland-Hawthorn, M. Englund, and G. Edvell, Opt. Express 12, 5902 (2004).

4. J. Bland-Hawthorn, A. Buryak, and K. Kolossovski, J. Opt. Soc. Am. A 25, 153 (2008).

5. J. Bland-Hawthorn, J. S. Lawrence, G. Robertson, S. Campbell, B. Pope, C. Betters, S. G. Leon-Saval, T. Birks, R. Haynes, N. Cvetojevic, and N. Jovanovic, Proc. SPIE 7735, 77350N (2010).

6. S. G. Leon-Saval, T. A. Birks, J. Bland-Hawthorn, and M. Englund, Opt. Lett. 30, 2545 (2005).

7. D. Noordegraaf, P. M. W. Skovgaard, M. D. Nielsen, and J. Bland-Hawthorn, Opt. Express 17, 1988 (2009).

8. D. Noordegraaf, P. M. W. Skovgaard, M. D. Nielsen, J. Bland-Hawthorn, R. Haynes, and J. Lægsgaard, Opt. Express 18, 4673 (2010).

9. T. A. Birks, A. Diez, J. L. Cruz, S. G. Leon-Saval, and D. F. Murphy, in Frontiers in Optics (Optical Society of America, 2010), paper FTuU1.

10. R. R. Thomson, T. A. Birks, S. G. Leon-Saval, A. K. Kar, and J. Bland-Hawthorn, Opt. Express 19, 5698 (2011).

11. I. Spaleniak, N. Jovanovic, S. Gross, M. Ireland, J. S. Lawrence, and M. Withford, in Proceedings of the IEEE Conference on Lasers and Electro-Optics/Pacific Rim (IEEE, 2011), paper 5410-CT-5.

12. Vytran, http://www.vytran.com.

13. S. G. Leon-Saval, A. Argyros, and J. Bland-Hawthorn, Opt. Express 18, 8430 (2010).

14. D. Gloge, Appl. Opt. 10, 2252 (1971).

15. S. C. Ellis, J. Bland-Hawthorn, J. S. Lawrence, J. Bryant, R. Haynes, A. Horton, S. Lee, S. G. Leon-Saval, H.-G. Löhmannsröben, J. Mladenoff, J. O'Byrne, W. Rambold, M. Roth, and C. Trinh, Proc. SPIE 7735, 773516 (2010). 